\title{
AXISYMMETRIC RESPONSE OF A FLUID-FILLED SPHERICAL SHELL IN FREE VIBRATIONS*†
}

\author{
A. E. ENGIN \\ Highway Safety Research Institute, \\ The University of Michigan, Ann Arbor, Mich. 48105, U.S.A. \\ and \\ Y. KING LIU \\ Department of Engineering Mechanics, \\ The University of Michigan, Ann Arbor, Mich. 48104. U.S.A.
}

\begin{abstract}
This paper is concerned with a theoretical model of the head. Neuroanatomical and analytical considerations lead to a fluid-filled spherical shell as a first-generation model. The shell is considered thin, elastic, homogeneous, and isotropic. The shell equations include both membrane and bending effects in axisymmetric torsioniess motion. The motion of the fluid is governed by the wave equation. A free vibration analysis of the fluid-shell system is obtained in the form of a frequency equation. Compared with a fluid-filled rigid shell and an elastic shell in vacuo, the frequency spectrum of the fluid-shell system is almost a 'superposition' of the spectra of the two special cases. The exceptions appear as slight distortions in the neighborhood of the 'curve' intersections.
\end{abstract}

\section{INTRODUCTION}

IT Is a well-established fact that approximately three-quarters of the fatalities resulting from all accidents involve injury to the head, emphasizing the disproportionate vulnerability of this part of the human body. The mounting awareness of the gravity of the problem culminated in a Head Injury Conference in Chicago, 1965. Its Proceedings, edited by Caveness and Walker (1966), consist of 576 pages of contributions from practically every conceivable point of view concerning the problem of craniocerebral trauma.

The investigation presented here is part of a systematic study of the various mechanical properties of the head as revealed by its response to pressure waves. It is an attempt to theoretically model what is considered a very complicated situation with the hope of deducing from the analysis the most prominent features of craniocerebral trauma.

Section 2 delineates the various considerations leading to the choice of the analytical model. The model adopted is a thin, homogeneous, isotropic and elastic spherical shell containing an inviscid irrotational fluid (Fig. 1). The various limitations of the model are discussed and they form points of departure for future investigations. Section 3 summarizes previous studies in head injury and also investigations in mechanics which are similar to the adopted model. The formulation of the problem consisting of the governing partial differential equations and their associated initial and boundary conditions are given in Section 4. The steps leading up to the frequency equation and mode shapes are revealed in Section 5. Discussion and presentation of the results in Section 6 terminate this paper.

\section{CONSIDERATIONS LEADING TO THE ANALYTICAL MODEL}

\section{Geometry, hydrostatics and restraints}

Roughly speaking, the human head can be represented as a shell filled with fluid inside

\footnotetext{
*Received 30 June 1969.

tThis work is part of a dissertation submitted to the Department of Engineering Mechanics of the University of Michigan for the partial fulfillment of the requirements for a Ph.D. degree.

$\ddagger$ Visiting Assistant Professor of Aeronautics and Astronautics, Stanford University, Stanford, Calif. 94305, U.S.A. (1968-69).
} 


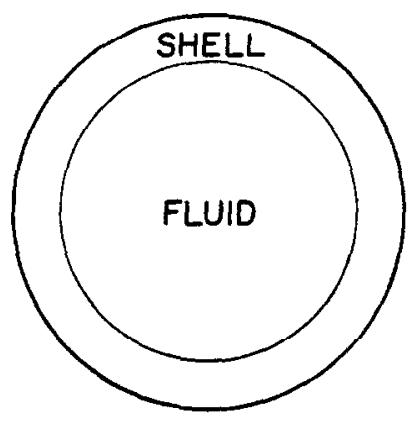

Fig. 1. Simplified model of the brain and skull as a fluidfilled spherical shell.

of which is a fluid mass of somewhat greater density. Consider Fig. 2 as an idealization of the problem. Region I represents the brain, II, the cerebrospinal fluid (CSF) and III, the

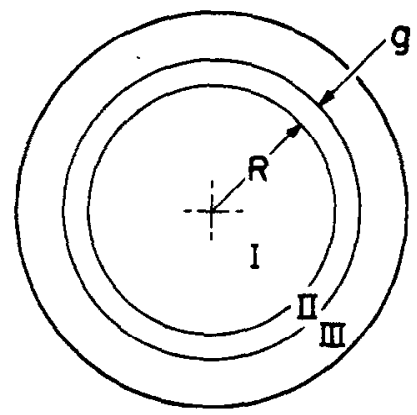

Fig. 2. Model of the brain, cerebrospinal fluid and skull.

skull. According to Blinkov and Glezer (1966), the cerebrospinal fluid has a specific weight of $1.007 \mathrm{~g} / \mathrm{cm}^{3}$ and the average adult $(20 \mathrm{yr}$ old) brain is $1200 \mathrm{~cm}^{3}$ in volume and $1378 \mathrm{~g}$ in weight and has a cranial (volume) capacity of $1500 \mathrm{~cm}^{3}$. Assuming spherical regions, one can estimate the dimensions involved:

$(4 / 3) \pi R^{3}=1200 \mathrm{~cm}^{3}$;

$$
R=(206.90)^{1 / 3} \cong 5.92 \mathrm{~cm} .
$$

The gap size, $g$, is, similarly:

$$
(4 / 3) \pi(R+g)^{3}=1500 \mathrm{~cm}^{3} ; g=4.57 \mathrm{~mm}
$$

McRae (1966) indicated a closed fit of the brain and skull.

From the well-known buoyancy relationship of hydrostatics we can find the weight of the brain in the CSF, $W_{\mathrm{CSF}}$, as the difference between its weight in air, $W_{\text {air }}$, and its buoyant force:

$$
\begin{aligned}
W_{\mathrm{CSF}} & =W_{\text {air }}-\gamma_{\mathrm{CSF}} V_{\text {brain }} \\
& =1378-(1.007)(1200) \cong 160 \mathrm{~g},
\end{aligned}
$$

where $\gamma$ and $V$ denote the specific weight and volume respectively. The above result conflicts with that given by Ganong (1967), who claims $W_{\mathrm{CSF}}=50 \mathrm{~g}$. The discrepancy can be explained only if the CSF does not completely enclose the brain. The level of the CSF which will agree with the Ganong figure can be computed from elementary hydrostatics. McRae (1966) asserts that more of the CSF is at the bottom of the head than it is on top in the normal position.

That the restraint system should be 'weak' can also be argued as follows. If the brain were not in relative hydrostatic equilibrium then there should exist 'strong' restraints in order to offset the density differences between the brain substance and the CSF. For example, in the normal upright position the brain should be prevented from sinking. Further, the restraint systems should be active whether one is upright, supine, lying prone or standing on one's head. A restraint system which is strong with respect to all these positional changes must be quite complicated. On the other hand, if the brain were in relative neutral equilibrium with the CSF, one need only 'weak' restraints to position it. We assume that nature follows the latter - the simpler of the two systems.

The quantitative estimates given above are supported by neuroanatomy. Figure 3 shows the major sub-divisions of the brain, brain stem and spinal cord. We note that the system is an integral unit and is also 'enclosed' in CSF, Davson (1960). The CSF fills the cerebral ventricles and the subarachnoid 


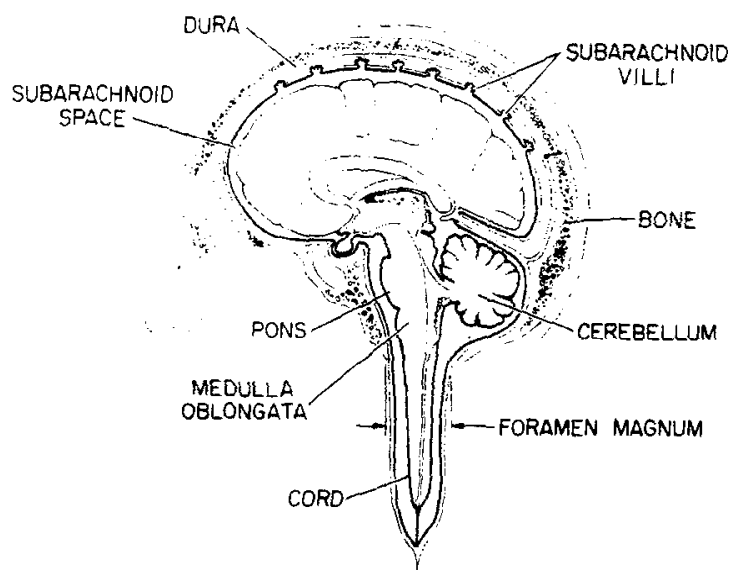

Fig. 3. Major organs of the carniocerebral system.

space. Figure 4 shows an enlarged section of the brain and its investing membranes. We quote from Ganong (1967), "The dura is attached firmly to bone. There is normally no 'subdural space', the arachnoid being held to the dura by the surface tension of the thin layer of fluid between the 2 membranes. The brain itself is supported within the arachnoid by the blood vessels and nerve roots and by multiple, fine fibrous arachnoid trabeculae. The brain weighs about $1400 \mathrm{~g}$ in air, but in its 'water bath' of CSF it has a net weight of only $50 \mathrm{~g}$. The buoyancy of the brain in the
CSF permits its relatively fimsy attachments to suspend it very effectively. When the head receives a blow, the arachnoid slides on the dura and the brain moves, but its motion is gently checked by the CSF cushion and by the arachnoid trabeculae".

The view that the skull is a closed spherical shell is, of course, substantially in error. Its lack of sphericity is evident by inspection. The shell has a major opening called the foramen magnum, through which the medulla oblongata merges with the spinal cord, Fig. 3. Other openings such as the optic foramen are so small as to be negligible.

Furthermore, the head is not a free-floating object, as is implied in our model, but is pivoted about the atlas and its orientation maintained by muscles and ligaments of various sorts.

\section{Dynamical implications of above}

The geometry, hydrostatics and restraint system seem, at least, to call for a multilayered spheroidal shell (skull and membranes) with a weakly plugged hole (medulla oblongata in the foramen magnum) containing a 'fluid' (brain) partially suspended in another.

The complications can be removed if one

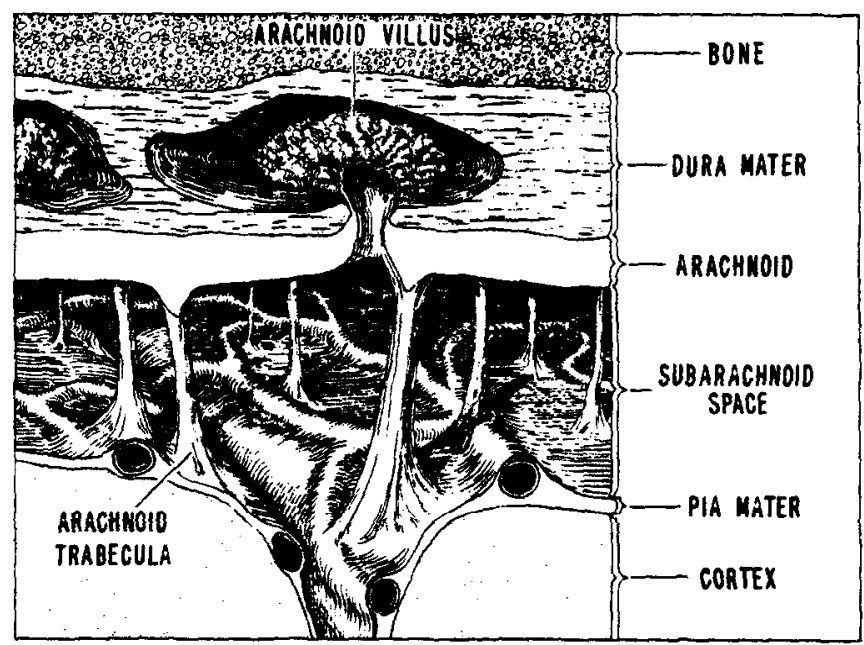

Fig. 4. Details of cerebral cortex and its investing membranes. 
takes into account experimental evidence and ultimately what one's objectives are. In the present case, we are interested primarily in the intracranial pressure distribution and the locations of high stress on the skull with the advent of a blow, i.e. a transient problem.

Roberts, Hodgson and Thomas (1966) conducted experiments with fluid-filled skulls subjected to different blows. They showed that the retension of falx cerebri and tentorium cerebelli membranes had practically no effect on the intracranial pressure distribution. Further, the simulation of an elastic closure over the foramen magnum produced changes which are different when compared to a rigid plug over the same. These results are probably reasonable since the material plugging over the foramen magnum in vivo are quite soft compared to skull bone. The assumption of a closed spherical shell is the most debatable and this point should be examined in the future.

While we recognize local variations in the brain, it should not be forgotten that the brain consists mostly of water and has a specific weight of $1.15 \mathrm{~g} / \mathrm{cm}^{3}$. Hence, a fluid-filled closed spherical shell appears to be a reasonably adequate first approximation since the densities of CSF and brain are approximately the same.

The primary value of the present model lies in providing a basis for later, more accurate, analyses: e.g. the inclusion of a softly plugged hole to simulate the foramen magnum. As the present model is already a complex and interesting problem in applied mechanics, we feel its analysis could be useful in guiding future investigations in the dynamic behavior of the head during impact.

\section{PREVIOUS STUDIES}

Previous work in the area can be put into two categories: (a) those which dealt directly with the analytical modelling of the head injury problem and (b) those which are investigations in theoretical mechanics as problems in fluidsolid interaction.
Category (a) has been treated by Anzelius (1943) and Guittinger (1950). They considered the effect of a blow to a free-floating rigid closed spherical shell with irrotational, inviscid fluid inside. Their formulations were practically identical and involved an axisymmetric solution of the wave equation in spherical coordinates. They concluded that the initial velocity input produced a compression wave at the point of impact (coup); however, because the shell was assumed rigid, the effect was instantaneously transmitted to the counterpole (contrecoup), whereupon a tension wave was simultaneously emitted. The collision of the two waves at the center produced large pressure gradients, which was considered the mechanism of damage. The obvious defect in the model led Goldsmith (1966) to suggest the analytical or numerical solution of a fluid-filled elastic shell. Goldsmith's paper is both a tutorial (for those not in the physical sciences) and a review of the theoretical and experimental approaches employed in engineering impact which might have implications in the delineation of head injury problem.

Recently, Hayashi (1968) treated a onedimensional version of tha Anzelius-Guittinger model. The system consists of a rigid but massless vessel (skull) containing internal elastic fluid (brain and CSF). The vessel is attached to a linear spring, which represents the composite elastic properties of the helmet, skull, skin, hair and the elasticity of the wall. Thus the problem is simplified to that of a fluid 'rod' enclosed in a rigid vessel with an attached spring striking a stationary wall. Approximate solutions were obtained for the extreme cases of very soft and very hard impacts. The analytical results agreed quite well with the experimental data of Roberts et al. (1966) and Fujii and Kobayashi (unpublished). The simple model has the advantage of being easy to interpret. However, many shortcomings are immediately evident: (a) it is not clear what occurs when the impact is neither very soft nor very hard; (b) due to 
the geometrical assumption, there is no way of determining the possible locations of skull fracture and (c) the effects of skull deformation on the intracranial pressure or pressure gradient cannot be determined. Both theoretical and experimental considerations indicate the desirability of a systematic study of the dynamic characteristics of a fluid-filled elastic spherical shell.

The investigations belonging to the second category are numerous. Only a few representative one's will be mentioned here. The problem of a fluid-filled shell submerged in another fluid has attracted many researchers, especially in the discipline of acoustics. Junger (1952) calculated both the refiection from an air-filled shell submerged in a fluid and the transmission through the shell interior of an incidental plane wave. Greenspon (1961) treated unpressurized shells by exact elasticity theory and the cylindrical shells with internal fluid by approximate shell theory. Goodman and Stern (1962), using elasticity theory and numerical integration of a system of ordinary differential equations, investigated the steady state response of fluid-filled spherical shell submerged in another fluid. Hickling (1962) extended the results to a pressure pulse emanating from a point source. The transient response to such an impulsive pressure can be found by integrating, over a suitable range of frequencies, the product of the steady-state response and the Fourier transform of the applied impulsive pressure. Recently, Rand and DiMaggio (1967) obtained frequency equations and mode shapes for the axisymmetric, extensional, torsionless vibrations of fluid-filled elastic spherical shells and rigid prolate spheroidal shells. As a problem in mechanics, the present investigation is a generalization of the results of Rand and DiMaggio by including the hending effect.

There exists two other areas where the dynamics of fluid-filled (cylindrical) shells have been extensively investigated: (a) the interaction of blood with arteries and/or veins and (b) the interaction of liquid pro- pellants and their elastic containers. The references given in Skalak (1966) in the first area and Beam and Guist (1967) in the second suffice to indicate the scope and interest in these two neighboring disciplines.

\section{FORMULATION OF THE PROBLEM}

The model of the head under consideration is that of a fluid-filled closed spherical shell under axisymmetric torsionless motion. The fluid is inviscid and irrotational while the shell is thin and elastic. The spherical coordinate system used is shown in Fig. 5.

Deformation of a given shell can be analyzed in terms of the deformation of its middle surface, i.e. a surface which lies midway between the thickness of the shell. The symbols $u, v$ and $w$ are used to denote the meridional $(\varphi)$, circumferential $(\theta)$ and radial $(r)$ displacements, respectively. Following the thin shell theory of Novozhilov (1960), we note that the assumptions of axisymmetry and torsionless displacements result in

$$
\frac{\partial \square}{\partial \theta}=0 ; v=0,
$$

where $\square$ denotes any function of the dependent variable. The remaining displacements are now expressible as:

$$
u=u(\varphi, t) ; w=w(\varphi, t) .
$$

The motion of an inviscid and irrotational fluid undergoing small oscillations is governed by the wave equation. In spherical coordinates it can be shown to be: (see Ewing, Jardetsky and Press, 1957)

$$
\begin{aligned}
& \frac{1}{r^{2}} \frac{\partial}{\partial r}\left(r^{2} \frac{\partial \phi}{\partial r}\right)+\frac{1}{r^{2} \sin \varphi} \frac{\partial}{\partial \varphi} \\
& \times\left(\sin \varphi \frac{\partial \phi}{\partial \varphi}\right)=\frac{1}{c^{2}} \frac{\partial^{2} \phi}{\partial t^{2}}
\end{aligned}
$$

where $\phi$ is the velocity potential and $c$ is the speed of sound in the fluid. The pressure, $P$, in 
the fluid is found from the velocity postential $\phi$, by the relationship:

$$
P=-\rho_{f}(\partial \phi / \partial t)
$$

where $\rho_{f}$ is the density of the fluid.

The equations of motion for the fluid-filled shell can now be derived from Hamilton's Principle. The potential energy $V$, of the shell is:

$V=\int_{S} \bar{U} \mathrm{~d} S-\int_{S}\left(P(a, \varphi, t)+P_{e}\right) w \mathrm{~d} S$

where the first integral represents the strain energy of the deformed shell, ( $\bar{U}$ is the strain energy density per unit area of mid-surface), the second integral represents the potential energy contribution due to effects of both the internal pressure $P(a, \varphi, t)$ and external pressure $\boldsymbol{P}_{e}$. The kinetic energy of the shell is

$$
T=\frac{1}{2} \int \mathbf{V}_{s} \rho_{s}\left[\left(\frac{\partial u}{\partial t}\right)^{2}+\left(\frac{\partial w}{\partial t}\right)^{2}\right] \mathrm{dV}_{s}
$$

where $\rho_{s}$ and $V_{d}$ are the mass density and the volume of the shell material respectively. In (7), the effects of shear deformation and rotary inertia of the shell are neglected.

The analytical statement of Hamilton's principle is

$$
\delta \int_{t_{1}}^{t_{2}}(T-V) \mathrm{d} t=0
$$

where $t_{1}$ and $t_{2}$ are two distinct, arbitrary, but fixed times, and $\delta$ denotes the usual variational operation. Substituting (6) and (7) into (8) and performing the variational operations yield two partial differential equations, (and a set of natural boundary conditions, which is of little importance here) i.e.

$$
\begin{gathered}
\left(1+\alpha^{2}\right)\left[-\frac{\partial^{2} u}{\partial \varphi^{2}}-\cot \varphi \frac{\partial u}{\partial \varphi}+\left(\nu+\cot ^{2} \varphi\right) u\right] \\
+\alpha^{2} \frac{\partial^{3} w}{\partial \varphi^{3}}+\alpha^{2} \cot \varphi \frac{\partial^{2} w}{\partial \varphi^{2}}
\end{gathered}
$$

$$
\begin{aligned}
& -\left[\alpha^{2}\left(\cot ^{2} \varphi+\nu\right)+(1+\nu)\right] \frac{\partial w}{\partial \varphi} \\
& +\frac{1-\nu^{2}}{E} \rho_{\&} a^{2} \frac{\partial^{2} u}{\partial t^{2}}=0,
\end{aligned}
$$

$$
\begin{aligned}
\alpha^{2} \frac{\partial^{3} u}{\partial \varphi^{3}} & +2 \alpha^{2} \cot \varphi \frac{\partial^{2} u}{\partial \varphi^{2}} \\
& -\left[(1+\nu)\left(1+\alpha^{2}\right)+\alpha^{2} \cot ^{2} \varphi\right] \frac{\partial u}{\partial \varphi} \\
& +\left[\alpha^{2} \cot ^{3} \varphi+3 \alpha^{2} \cot \varphi\right. \\
& \left.-(1+\nu)\left(1+\alpha^{2}\right) \cot \varphi\right] u \\
& -\alpha^{2}\left[\frac{\partial^{4} w}{\partial \varphi^{4}}+2 \cot \varphi \frac{\partial^{3} w}{\partial \varphi^{3}}\right. \\
& -\left(1+\nu+\cot ^{2} \varphi\right) \frac{\partial^{2} w}{\partial \varphi^{2}} \\
& \left.+\left(2 \cot \varphi+\cot ^{3} \varphi-\nu \cot \varphi\right) \frac{\partial w}{\partial \varphi}\right] \\
& -2(1-\nu) w-\frac{1-\nu^{2}}{E} \rho_{s} a^{2} \frac{\partial^{2} w}{\partial t^{2}} \\
& -\frac{1-\nu^{2}}{E h} \alpha^{2}\left[\rho_{f} \frac{\partial \phi(a, \varphi, t)}{\partial t}-P_{e}(\varphi, t)\right]=0
\end{aligned}
$$

where $E$ is the modulus of elasticity, $a$ is the inner radius, $h$ is the thickness of the shell, $\nu$ is the Poisson's ratio and $\alpha^{2}=h^{2} / 12 a^{2}$ is a thickness parameter of the shell.

Equations (4), (9) and (10) are the governing partial differential equations of the problem. The velocity potential $\phi$ and the radial displacement $w$ are interconnected through the kinematic boundary condition, i.e. the radial velocities of the shell and fiuid are equal for all $\varphi$ and $t$, or

$$
\frac{\partial w}{\partial t}(\varphi, t)=\frac{\partial \phi}{\partial r}(a, \varphi, t)
$$

\section{FREQUENCY EQUATION AND MODE SHAPE}

For no external pressure pulse, i.e. $P_{e}(\varphi)=$ 0 , we obtain the free vibration response of the fluid-shell system. Introduce the dimensionless variables: 


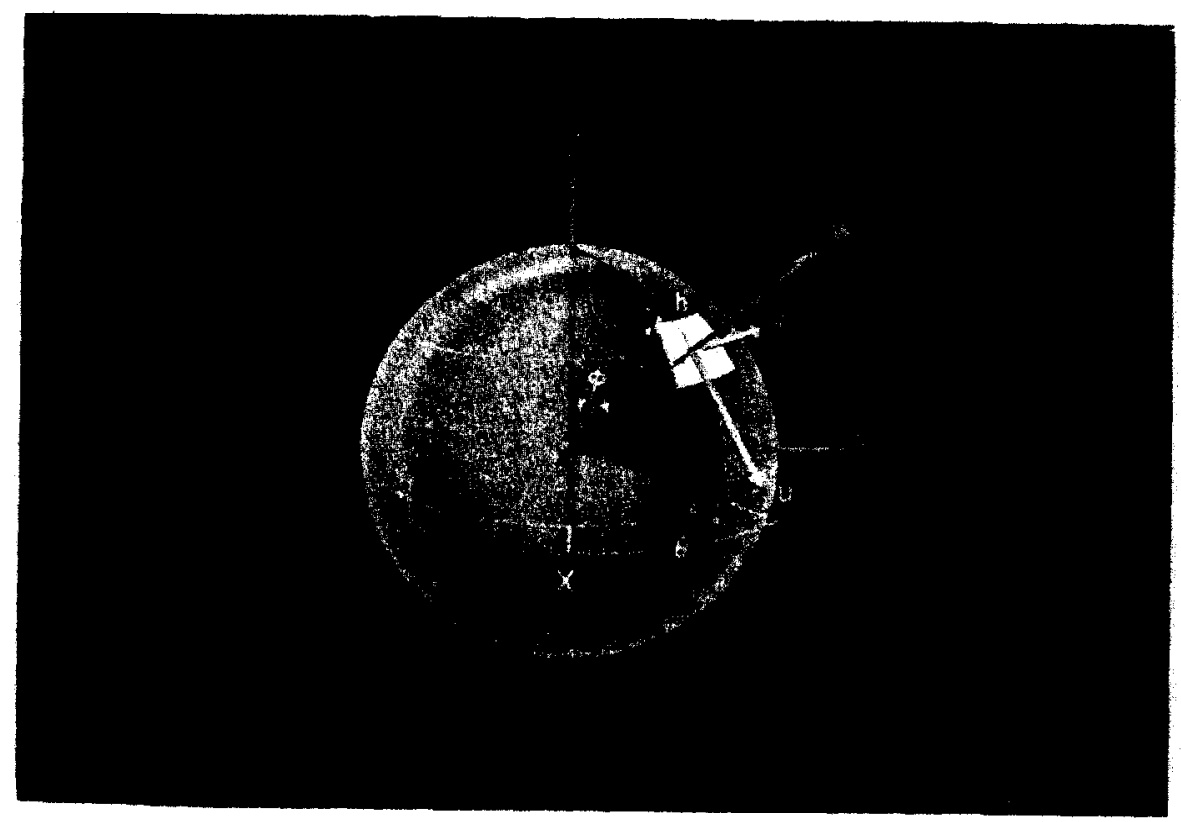

Fig. 5. Spherical polar coordinate system.

(facing $p .16$ ) 


$$
\begin{aligned}
& \begin{array}{l}
\psi \\
=u / a, \zeta=w / a \\
\tau=c_{s} t / a, c_{s}=\left[E / \rho_{s}\left(1-\nu^{2}\right)\right]^{1 / 2}
\end{array} \\
& s=c / c_{s}, r_{1}=r / a, \Phi_{1}=\phi / a c_{s}, f=\rho_{f} a / \rho_{s} h
\end{aligned}
$$

where $c_{B}$ is the apparent wave speed in the shell (symmetrical wave speed in an infinite plate) and $f$ is the fluid-shell interaction parameter.

Equations (4), (9), (10) and (11) become, in view of (12):

$$
\begin{aligned}
\frac{1}{r_{1}^{2}} \frac{\partial}{\partial r_{1}}\left(r_{1}{ }^{2} \frac{\partial \Phi_{1}}{\partial r_{1}}\right) & +\frac{1}{r_{1}^{2} \sin \varphi} \frac{\partial}{\partial \varphi} \\
& \times\left(\sin \varphi \frac{\partial \Phi_{1}}{\partial \varphi}\right)-\frac{1}{s^{2}} \frac{\partial^{2} \Phi_{1}}{\partial \tau^{2}}=0 \\
\alpha^{2}\left[\frac{\partial^{2} \psi}{\partial \varphi^{2}}+\right. & \cot \varphi \frac{\partial \psi}{\partial \varphi}-\left(\nu+\cot ^{2} \varphi\right) \psi-\frac{\partial^{3} \zeta}{\partial \varphi^{3}} \\
& \left.-\cot \varphi \frac{\partial^{2} \zeta}{\partial \varphi^{2}}+\left(\nu+\cot ^{2} \varphi\right) \frac{\partial \zeta}{\partial \varphi}\right] \\
& +\frac{\partial^{2} \psi}{\partial \varphi^{2}}+\cot \varphi \frac{\partial \psi}{\partial \varphi}-\left(\nu+\cot ^{2} \varphi\right) \psi \\
& +(1+\nu) \frac{\partial \zeta}{\partial \varphi}-\frac{\partial^{2} \psi}{\partial \tau^{2}}=0 \\
\alpha^{2}\left[\frac{\partial^{3} \psi}{\partial \varphi^{3}}+\right. & 2 \cot \varphi \frac{\partial^{2} \psi}{\partial \varphi^{2}}-\left(1+\nu+\cot ^{2} \varphi\right) \frac{\partial \psi}{\partial \varphi} \\
& +\cot \varphi\left(2-\nu+\cot ^{2} \varphi\right) \psi-\frac{\partial^{4} \zeta}{\partial \varphi^{4}} \\
& -\frac{\partial^{2} \zeta}{\partial \tau^{2}}-f \frac{\partial \Phi}{\partial}(1, \varphi, \tau) \\
& -2 \cot \varphi \frac{\partial^{3} \zeta}{\partial \varphi^{3}}+\left(1+\nu+\cot ^{2} \varphi\right) \frac{\partial^{2} \zeta}{\partial \varphi^{2}} \\
& \left.-\cot \varphi\left(2-\nu+\cot ^{2} \varphi\right) \frac{\partial \zeta}{\partial \varphi}\right] \\
& (1+\nu)\left(\frac{\partial \psi}{\partial \varphi}+\cot _{\varphi} \psi\right.
\end{aligned}
$$

Using the method of separation of variaables on (13), i.e. assume $\Phi_{1}\left(r_{1}, \varphi, \tau\right)=$
$R\left(r_{1}\right) G(\varphi) e^{i \Omega s t}$, we obtain two ordinary differential equations:

$$
\begin{gathered}
\frac{1}{\sin \varphi} \frac{d}{d \varphi}\left(\sin \varphi \frac{d G}{d \varphi}\right)+\lambda G=0 \\
\frac{d}{d r_{1}}\left(r_{1}^{2} \frac{d R}{d r_{1}}\right)+\left(\Omega^{2} r_{1}^{2}-\lambda\right) R=0
\end{gathered}
$$

where $\Omega=w a / c$ is the unknown dimensionless frequency and $\lambda$ is a constant. Equation (17) is the self-adjoint from of Legendre's equation. From orthogonal function theory, e.g. Churchill (17) will have a Legendre polynomial solution if and only if $\lambda=n(n+1)$, where $n$ are integers. Equation (18) has as its solution the spherical Bessel functions. The requirement of boundedness and the linearity of (13) lead to its general solution:

$$
\Phi_{1}\left(r_{1}, \varphi, \tau\right)=\sum_{n=0}^{\infty} C_{n} j_{n}\left(\Omega r_{1}\right) P_{n}(\cos \varphi) \mathrm{e}^{i \Omega s r}
$$

when $C_{n}$ are unknown coefficients, $j_{n}\left(\Omega r_{1}\right)$ are spherical Bessel functions of the first kind and $P_{n}(\cos \varphi)$ are Legendre polynomials of the first kind.

Similar considerations lead to the following expansions for $\zeta$ and $\psi$ in (14) and (15):

$$
\begin{aligned}
\zeta(\varphi, t) & =\sum_{n=0}^{\infty} A_{n} P_{n}(\cos \varphi) \mathrm{e}^{i \Omega s t}, \\
\psi(\varphi, \tau) & =\sum_{n=1}^{\infty} B_{n} P_{n}{ }^{1}(\cos \varphi) \mathrm{e}^{i \Omega m}
\end{aligned}
$$

where $A_{n}$ and $B_{n}$ are coefficients and $P_{n}{ }^{1}(\cos \varphi)$ are Associated Legendre Polynomials of the first kind and first order. The boundary condition (16) leads to the relationship:

$$
C_{n}=i s A_{n} / j_{n}^{\prime}(\Omega)
$$

where' denotes differentiation with respect to its argument. Hence, (19) can be rewritten as:

$$
\begin{aligned}
\Phi_{1}\left(r_{1}, \varphi, \tau\right)= & \sum_{n=0}^{\infty} A_{n}\left[i s / j_{n}^{\prime}(\Omega)\right] \\
& \times j_{n}\left(\Omega r_{1}\right) P_{n}(\cos \varphi) \mathrm{e}^{i \Omega s \tau}
\end{aligned}
$$


The substitution of (20), (21) and (23) into (14) and (15) leads to two sets of homogeneous algebraic equations for $A_{n}$ and $B_{n}$; where the higher-order derivatives of $P_{n}$ and $P_{n}{ }^{1}$ have been eliminated by the recursive use of the differential equations which they satisfy. Requiring that nontrivial solutions exist for the coefficients $A_{n}$ and $B_{n}$ yields the frequency equations:

For $n=0$,

$$
\left[1+f \frac{j_{0}(\Omega)}{\Omega j_{0}^{\prime}(\Omega)}\right] s^{2} \Omega^{2}-2(1+\nu)=0
$$

For $n \geqslant 1$ and $\lambda_{n}=n(n+1)$

$$
\begin{aligned}
& {\left[1+f \frac{j_{n}(\Omega)}{\Omega j_{n}^{\prime}(\Omega)}\right] s^{4} \Omega^{4}+\left\{\left[1+f \frac{j_{n}(\Omega)}{\Omega j_{n}^{\prime}(\Omega)}\right]\right.} \\
& \quad \times\left(1-\nu-\lambda_{n}\right)\left(1+\alpha^{2}\right) \\
& \left.-2(1+\nu)-\alpha^{2}\left[\lambda_{n}^{2}-\lambda_{n}(1-\nu)\right]\right\} s^{2} \Omega^{2} \\
& -(1+\nu)\left\{2\left(1-\nu-\lambda_{n}\right)\left(1+\alpha^{2}\right)\right. \\
& \left.+\lambda_{n}\left[1+\nu-\alpha^{2}\left(1-\nu-\lambda_{n}\right)\right]\right\} \\
& -\alpha^{2}\left(2-\lambda_{n}\right)\left[\lambda_{n}^{2}-\lambda_{n}(1-\nu)\right]=0 .
\end{aligned}
$$

The appropriate limiting cases of the above frequency equations agree with results obtained by other authors.

\section{Case 1}

The case of $f=0$ corresponds to the absence of fiuid. Introduction of values of $s$ and $\Omega$ into (24) gives the dimensional angular frequency of pure radial motion as

$$
\omega_{0}=(1 / a)\left[2 E / \rho_{s}(1-\nu)\right]^{1 / 2}
$$

which was first obtained by Lamb (1882). Setting $f=0$ and defining a new nondimensional frequency $\Omega=\Omega s$ in (25) yields the frequency equation of the empty shell which was recently obtained by McIvor and Sonstegard (1966)

$$
\begin{aligned}
& \Omega^{4}-\left[1+3 \nu-\alpha^{2}(1-\nu)\right. \\
& \left.+\lambda_{n}\left(1+\nu \alpha^{2}\right)+\alpha^{2} \lambda_{n}{ }^{2}\right] \bar{\Omega}^{2} \\
& +\left[\alpha^{2} \lambda_{n}{ }^{3}-4 \alpha^{2} \lambda_{n}{ }^{2}+\alpha^{2} \lambda_{n}\left(5-\nu^{2}\right)+\lambda_{n}\left(1-\nu^{2}\right)\right. \\
& \left.-2\left(1+\alpha^{2}\right)\left(1-\nu^{2}\right)\right]=0
\end{aligned}
$$

\section{Case 2}

The case of $f>0$ and $s \rightarrow 0$ corresponds to a rigid shell containing a fluid.

The frequency equations (24) and (25) degenerate to

$$
j_{n}^{\prime}(\Omega)=0
$$

which is easily shown to be the same as Güttinger's (1950) result.

$$
2 \Omega J_{n+1 / 2}^{\prime}(\Omega)=J_{n+1 / 2}(\Omega)
$$

where $J_{n+1 / 2}(\Omega)$ is the Bessel function of the indicated order.

Case 3

The case of $\alpha^{2}=0$ yields the frequency equation corresponding to the membrane (extensional) theory for both the empty shell $(f=0)$ and fluid-filled shell $(f>0)$. The frequency equations for vibrations of a fluidfilled spherical membrane possessing infinite bulk modulus $(\nu=1 / 2)$ were given by Morse and Feshbach, and their results agree with (24) and (25) when $\alpha^{2}, f$ and $\nu$ are given the appropriate values.

Figure 6 is a plot of the frequency spectrum for a spherical shell in vacuo obtained from (27), using $\nu=0.3$ and $a / h=20$. Note that in all the plots the mode numbers $n$ are discrete, i.e. only those points corresponding to the integer values of $n$ are physically meaningful. In Fig. 6, both the composite* (lower branch) and the membrane mode (higher branch) frequencies are plotted using the nondimensional frequency $\bar{\Omega}$, and also $\Omega=\bar{\Omega} / s$. The value of $s$ for bone-water and steel-water are 0.553 and 0.269 respectively.

*This type of classification was used in Mclvor and Sonstegard (1966). 


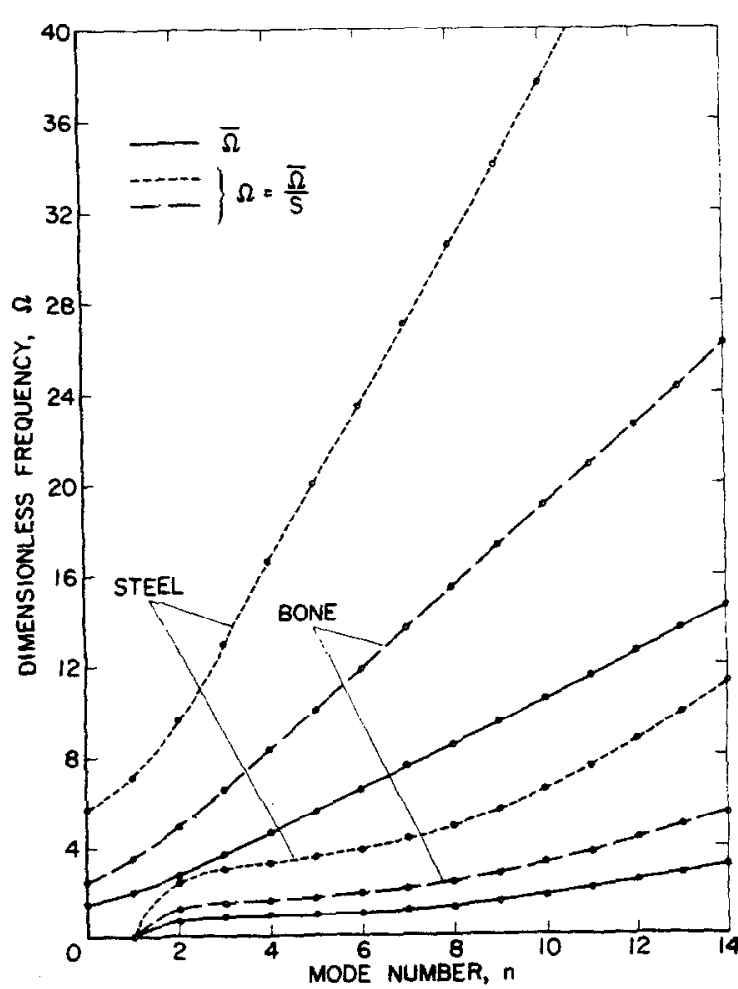

Fig. 6. Frequency spectrum for an elastic spherical shell in vacuo $(\nu=0.3, a / h=20, s=0.269$ for steel-water and $s=0.553$ for bone-water).

The reason for plotting the same frequency spectrum in terms of two nondimensional frequency parameters, namely $\bar{\Omega}$ and $\Omega$, will be readily seen in connection with Figs. 7-9.

Equation (28a), which gives the frequency spectrum for an ideal fluid in a rigid spherical shell, is plotted in Fig. 7. In Figs. 8 and 9, the frequency spectra corresponding to (24) and (25) are plotted for $\nu=0.3, a / h=20$ and $f$ is equal to 9.38 (bone-water) and 2.56 (steelwater) respectively.

\section{DISCUSSION OF RESULTS}

Three results reveal themselves from a comparison of Figs. 6 through 9: (a) In Figs. 6 and 7 . the frequency spectra represent the natural frequencies of an empty shell and a fluid-filled rigid shell, respectively. In Figs. 8 and 9 , one can no longer say that a particular frequency of the spectrum belongs to the shell

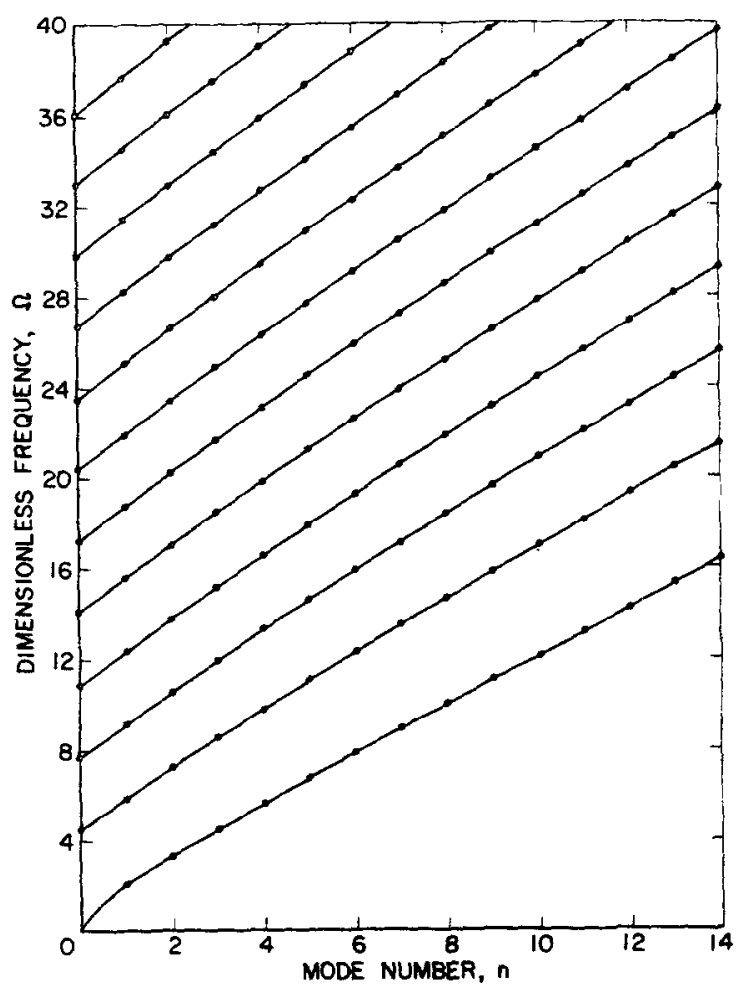

Fig. 7. Frequency spectrum for a rigid fluid-filled spherical shell.

or to the fluid, since each frequency is a natural frequency of the fluid-filled elastic spherical shell system.

(b) When the shell containing the fluid becomes elastic, certain portions of the spectrum become distorted. In Figs, 6 and 8 or 9 are superposed, the higher branches of $\Omega$ in Fig. 6 pass tangent to the distorted portion of the spectrum in Figs. 8 and 9. The higher branches in Fig. 6, however, are associated mainly with the membrane behavior of the shell. Hence, this 'higher-branch distortion' phenomenon is the result of the membrane behavior of the shell serving as an elastic boundary for the fluid. In other words, one of the predominant fluid-solid interactions in this problem is between the membrane action of the shell with the fluid. It is interesting to note from the trend exhibited in Figs. 8 and 9 that should the value of $f$ be increased further, 


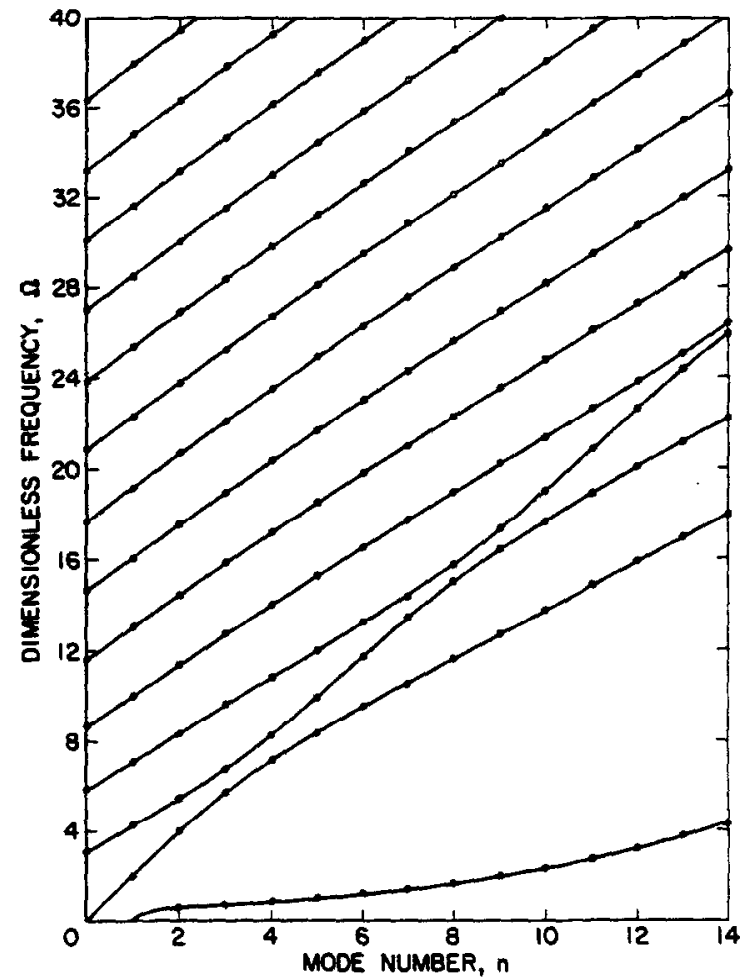

Fig. 8. Frequency spectrum for an elastic fluid-filled (bone) spherical shell. $\nu=0.3, f=9.38, s=0.553$.

i.e. the shell material becomes softer, the upper branch of Fig. 6 may become lower than the lowest branch of Fig. 7.

(c) Comparison of Figs. 8 or 9 with Fig. 7 exhibits a frequency branch which does not exist in the spectrum of the latter. This is the lowest branch of the frequencies displayed in Figs. 8 and 9. The existence of this lower branch' in the frequency spectrum for an elastic fluid-filled shell corresponds to the composite mode in Fig. 6 of the empty shell. The numerical values of the in-vacuo lower (composite) branch are changed due to the presence of the fluid, but qualitatively the branch remains similar. The composite mode behavior in the empty shell was explained by McIvor and Sonstegard (1966), as exhibiting membrane behavior for small $\mathbf{n}$ and bending behavior for large $\mathbf{n}$.

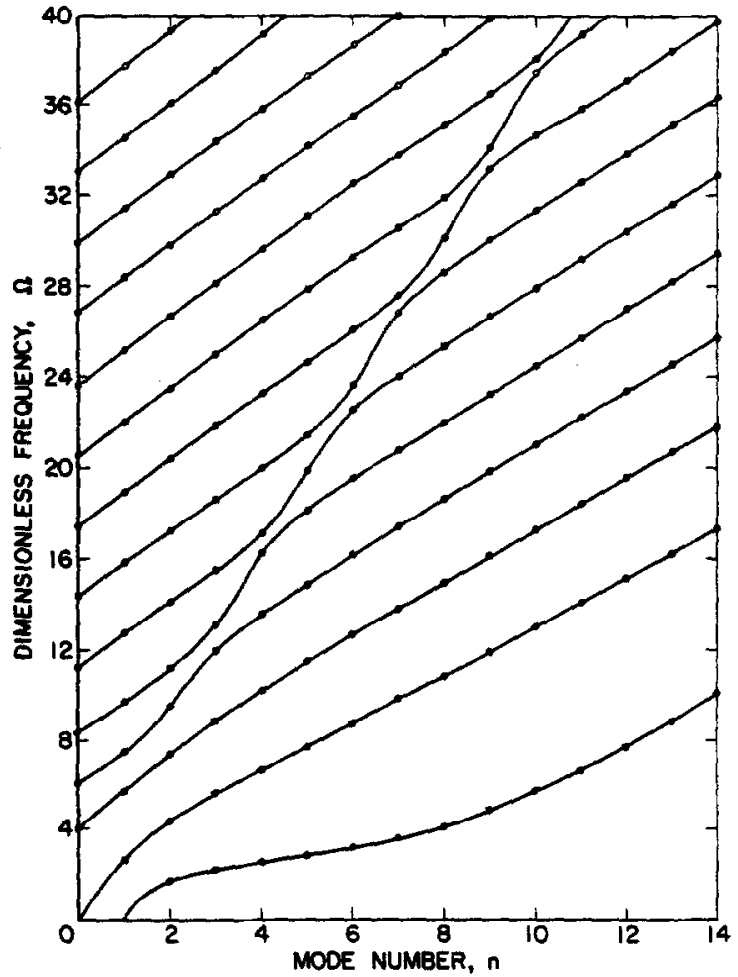

Fig. 9. Frequency spectrum for an elastic fiuid-filled (steel) spherical shell. $\nu=0.3, f=2 \cdot 56, s=0.269$.

Within the context of the present model some improvements and/or extensions can be made without drastically altering its basic makeup. Having obtained an elastic solution, one can appeal to the Correspondence Principle of Viscoelasticity to obtain viscoelastic solutions of the same problem in the spirit of Maxwell and Anliker (1968). The linearized equations for spherical shells shown in (9) and (10) might also be valid for 'thick' walls. Greenspon (1961) showed that the shell equations corresponding to (9) and (10) for the case of a cylindrical shell continues to be valid for so-called 'thick' walls. A thin elastic spherical shell emphasizes the membrane-fluid interaction, see Fig. 9. We might expect more participation by the bending modes for thick shell, i.e. shell with $a / h<$ 20. The response of the model to external 
pressure pulses must necessarily make use of the results given here. These results, however, will be presented in other papers.

Acknowledgements - The authors gratefully acknowledge the support of this research by the National Institute for Neurological Diseases and Blindness under Contract No. 43-67-1136 and the National Center for Urban and Industrial Health under Grant No. UI00025-02.

On aspects of neuroanatomy, we extend our appreciation to Drs. Webb Haymaker and Eric Ogden of the NASA Ames Research Center for their advice. Graphical assistance was also provided by the Center.

\section{REFERENCES}

Anzelius, A. (1943) The effect of an impact on a spherical liquid mass. Acta path. microbiol. scand. Suppl. 48, 153-159.

Beam, R. M. and Guist, L. R. (1967) The axially symmetric response of an elastic cylindrical shell partially filled with liquid. NASA Rep. No. TN D-38 77.

Blinkov, S. M. and Glezer, I. I. (1968) The Human Brain in Figures and Tables, p. 336. Plenum Press, New York.

Caveness, W. F. and Walker, A. E. (Editors) (1966) Head Injury. Lippincott, Philadelphia.

Churchill, R. V. (1963) Fourier Series and Boundary Value Problems, 2nd Edn., p. 203. McGraw-Hill, New York.

Davson, H. (1961) Intracranial and intraocular fluids. Handbook of Physiology, Parts 1-3, Chap. 122, p. 1761. American Physiological Society. New York.

Ewing, W. M., Jardetsky, W. S. and Press, F. (1957) Elastic Waves in Layered Media, p. 8, McGraw-Hill, New York.

Fujii, S. and Kobayashi, H. (1967) Unpublished Report of Jap. Soc. Auto Engrs.

Ganong, W. F. (1967) Review of Medical Physiology, 3rd Edn, Chap. 32, p. 486. Lange Medical Pub., Los Altos.

Goldsmith, W. (1966) The physical processes producing head injury. Head Injury, Chap. 29, pp. 350-382. Lippincott, Philadelphia.

Goodman, R. R. (1962) Reflection and transmission of sound by elastic spherical shells. J. acoust. Soc. Am. 34, 338-334.

Greenspon, J. E. (1961) Vibration of thick and thin cylindrical shells surrounded by water. $J$. acoust. Soc. Am. 33, 1321-1328.

Guttinger, W. (1950) Der Stosseffekt auf eine Flüssigkeitskugel als Grundlage einer Physikalischen Theorie der Etstehung von Gehirnverletzungen. $Z$. Naturf. 5 , 622-628.

Hayashi, T. (1968) Abs. I2th Int. Congr. Appl. Mech. and private communications.

Hickling, R. (1962) Analysis of echoes from a solid elastic sphere in water. J. acoust. Soc. Am. 34, 15821592.

Junger, M. C. (1952) Vibration of elastic shells in a fuid medium and associated radiation of sound. $J$. appl. Mech. 74, 439-445.

Lamb, H. (1882) On the vibration of spherical shell. Proc. Lond. math. Soc. 15, 50.

Maxwell, J. A. and Anliker, M. (1968) Dissipation and distension of small waves in arteries in veins with viscoelastic wall properties. Biophys. J. 8, 920-950.

Mclvor, I. K. and Sonstegard, D. A. (1966) Axisymmetric response of a closed spherical shell to a nearly uniform radial impulse. J. acoust. Soc. Am. 40, 1540-1547.

McRae, D. L. (1966) Proc. Head Injury Conf. Ref. I, Discussion, p. 523. Lippincott, Philadelphia.

Morse, P. M. and Feshbach, H. (1953) Methods of Theoretical Physics, Part 2. pp. 1469-1472. McGrawHill, New York.

Novozhilov, V. V. (1964) Thin Shell Theory, 2nd rev. Edn. Noordhoff, Holland.

Rand, R. and Dimaggio, F. (1967) Vibrations of fiuidfilled spherical and spheroidal shells. $J$. acoust. Soc. Am. 42. 1278-1286.

Roberts, V., Hodgson, V. and Thomas, L. M. (1967) Fluid pressure gradients caused by impact to the human skull. Biomechanics Mono. pp. 223-225. ASME, New York.

Skalak, R. and Fung, Y. C. (Editor) (1966) Proc. Biomechanics Symp. Am. Soc. mech. Engr pp. 20-46.

\section{NOMENCLATURE}

CSF cerebrospinal fluid

$E$ Young's modulus

$P_{e}$ external force distribution on the shell

$P_{n}(\cos \varphi) \quad$ Legendre polynomials of the first kind

$P_{n}{ }^{1}(\cos \varphi)$ associated Legendre polynomials of the first kind and first order

$R$ radius of the brain

$S$ mid-surface of the shell

$T$ kinetic energy

$V$ potential energy

$V_{s}, V_{s}$ volumes of fluid and shell respectively

$U$ strain energy density for the shell

$\phi$ velocity potential for the fiuid

$\Phi_{1}$ nondimensional velocity potential for the fluid, $\phi / a c_{z}$.

$\Omega$ nondimensional frequency, wa/c

$\Omega a$ radius of spherical shell

$A_{0}, A_{n}$ coefficients of the Legendre polynomial expansion of $\zeta$

$B_{n}$ coefficients of the Legendre polynomial expansion of $\psi$

$C_{0}, C_{n}$ coefficients of velocity potential

$c$ compressional wave speed in the fluid

$c$ apparent wave speed in the shell, $\left[E / \rho_{,}(1-\right.$ $\left.\left.v^{2}\right)\right]^{1 / 2}$

$f$ shell-fluid interaction parameter, $\rho_{0} a / \rho_{g} h$

$g$ cerebrospinal fiuid layer thickness

$h$ shell thickness

$j_{n}(z)$ spherical Bessel function, $(\pi / 2 z)^{1 / 2} J_{n+1 / 2}(z)$

$P(a, \varphi, t)$ fluid pressure on the surface of the shell

$r, \theta, \varphi$ spherical coordinates

$r_{1}$ nondimensional radius, $r / a$

$s$ speed ratio, $c / c_{\text {s }}$ 


\author{
$t$ time \\ $u$ meridional displacement with respect to \\ center of mass of the system \\ $w$ radial displacement with respect to center of \\ mass of the system \\ $x, y, z$ cartesian coordinates \\ $\alpha^{2}$ thickness parameter, $h^{2} / 12 a^{2}$ \\ $\delta$ variation symbol
}

$\zeta$ nondimensional radial displacement, $w / a$

$\psi$ nondimensional meridional displacement, $u / a$

$\lambda_{n}=n(n+1)$, where $n$ are integers

$\nu$ Poisson's ratio

$\rho_{f}, \rho_{\varepsilon}$ mass density of fluid and shell, respectively

$\tau$ nondimensional time, $c t / a$

$\omega$ angular frequency. 